Nikhef-2011-026

\title{
Effective Lifetimes of $B_{s}$ Decays and their Constraints on the $B_{s}^{0}-\bar{B}_{s}^{0}$ Mixing Parameters
}

\author{
Robert Fleischer and Robert Knegjens \\ Nikhef, Science Park 105, NL-1098 XG Amsterdam, The Netherlands
}

\begin{abstract}
Measurements of the effective lifetimes of $B_{s}$-meson decays, which only require untagged rate analyses, allow us to probe the width difference $\Delta \Gamma_{s}$ and the CPviolating phase $\phi_{s}$ of $B_{s}^{0}-\bar{B}_{s}^{0}$ mixing. We point out that the dependence of the effective lifetime on non-linear terms in $\Delta \Gamma_{s}$ allows for a determination of $\phi_{s}$ and $\Delta \Gamma_{s}$ given a pair of $B_{s}$ decays into $\mathrm{CP}$-even and CP-odd final states. Using recent lifetime measurements of $B_{s}^{0} \rightarrow K^{+} K^{-}$and $B_{s}^{0} \rightarrow J / \psi f_{0}(980)$ decays, we illustrate this method and show how it complements the constraints in the $\phi_{s}-\Delta \Gamma_{s}$ plane from other observables.
\end{abstract}

September 2011 



\section{Introduction}

A promising avenue for New Physics (NP) to enter the observables of $B_{s}$-meson decays is given by $B_{s}^{0}-\bar{B}_{s}^{0}$ mixing. In the Standard Model (SM), the phenomenon of mixing originates from box topologies and is strongly suppressed. In the presence of NP, new particles could give rise to additional box topologies or even contribute at the tree level. Should these NP contributions also involve new CP-violating phases, the $B_{s}^{0}-\bar{B}_{s}^{0}$ mixing phase $\phi_{s}$ could differ sizably from the tiny SM value of $-2.1^{\circ}$ (see, for instance, Refs. [1,2] and references therein).

A key channel for addressing this exciting possibility is $B_{s}^{0} \rightarrow J / \psi \phi$. A characteristic feature of this channel is that its final state contains two vector mesons and thereby requires a time-dependent angular analysis of the $J / \psi \rightarrow \mu^{+} \mu^{-}$and $\phi \rightarrow K^{+} K^{-}$decay products [2, 3]. Over the last couple of years, measurements at the Tevatron of CPviolating asymmetries in "tagged" analyses (distinguishing between initially present $B_{s}^{0}$ or $\bar{B}_{s}^{0}$ mesons) of the $B_{s}^{0} \rightarrow J / \psi \phi$ channel indicate possible NP effects in $B_{s}^{0}-\bar{B}_{s}^{0}$ mixing [4 6]. These results are complemented by the measurement of the anomalous like-sign dimuon charge asymmetry at $\mathrm{D} \varnothing$, which was found to differ by $3.9 \sigma$ from the SM prediction [7]. This summer, the LHCb collaboration has also joined the arena, reporting, however, results that disfavour large NP effects [8]. The above measurements, which we will discuss in more detail below, are typically shown in the $\phi_{s}-\Delta \Gamma_{s}$ plane, where $\Delta \Gamma_{s}$ is the width difference between the mass eigenstates of the $B_{s}$-meson system.

In this paper, we point out a new method for determining further constraints in the $\phi_{s}-\Delta \Gamma_{s}$ plane using measurements of the effective lifetimes of $B_{s}$ decays. In particular, we show that the information provided by the lifetimes of a pair of decays into CP-even and $\mathrm{CP}$-odd final states is sufficient to determine $\phi_{s}$ and $\Delta \Gamma_{s}$. The advantage of this strategy is that it only requires an "untagged" analysis, i.e. it is not necessary to distinguish between initially present $B_{s}^{0}$ or $\bar{B}_{s}^{0}$ mesons, which is experimentally advantageous.

Specifically, we will consider the $B_{s}^{0} \rightarrow K^{+} K^{-}\left[9,10\right.$ and $B_{s}^{0} \rightarrow J / \psi f_{0}(980)$ [11, 12 decays, which have final states with the $\mathrm{CP}$ eigenvalues +1 and -1 , respectively. From here on we shall abbreviate the latter decay as $B_{s}^{0} \rightarrow J / \psi f_{0}$. First measurements of the effective lifetimes of these channels are already available from the CDF and LHCb collaborations $[13-15]$. For the theoretical interpretation of these results we also need to address hadronic uncertainties. A closer look will reveal that these decays are well suited in this respect. We will illustrate our method with the most recent data and shall compare the resulting constraints in the $\phi_{s}-\Delta \Gamma_{s}$ plane with those from the alternative measurements listed above.

The outline is as follows: in Section 2, we discuss the general formalism to calculate effective lifetimes and show in Section 3 how the corresponding measurements can be converted into contours in the $\phi_{s}-\Delta \Gamma_{s}$ plane. In Section 4, we turn to the hadronic uncertainties affecting this analysis and their control through experimental data. The constraints on the $B_{s}^{0}-\bar{B}_{s}^{0}$ mixing parameters arising from the current data for the effective lifetimes of the $B_{s}^{0} \rightarrow K^{+} K^{-}$and $B_{s}^{0} \rightarrow J / \psi f_{0}$ channels are explored in Section 5 . where we also illustrate the impact of future lifetime measurements with errors at the $1 \%$ level. In Section 6, we give a collection of additional $B_{s}$ decays that can be added to this analysis in the future. Finally, we summarize our conclusions in Section 7. 


\section{General Formalism}

We will consider a $B_{s} \rightarrow f$ transition with a final state $f$ into which both a $B_{s}^{0}$ and a $\bar{B}_{s}^{0}$ meson can decay. The corresponding untagged rate can then be written as follows [2]:

$$
\begin{aligned}
\left\langle\Gamma\left(B_{s}(t) \rightarrow f\right)\right\rangle & \equiv \Gamma\left(B_{s}^{0}(t) \rightarrow f\right)+\Gamma\left(\bar{B}_{s}^{0}(t) \rightarrow f\right) \\
& =R_{\mathrm{H}}^{f} e^{-\Gamma_{\mathrm{H}}^{(s)} t}+R_{\mathrm{L}}^{f} e^{-\Gamma_{\mathrm{L}}^{(s)} t}
\end{aligned}
$$

where $\mathrm{L}$ and $\mathrm{H}$ denote the light and heavy $B_{s}$ mass eigenstates, respectively. Using

$$
\Gamma_{s} \equiv \frac{\Gamma_{\mathrm{L}}^{(s)}+\Gamma_{\mathrm{H}}^{(s)}}{2}=\tau_{B_{s}}^{-1}, \quad \Delta \Gamma_{s} \equiv \Gamma_{\mathrm{L}}^{(s)}-\Gamma_{\mathrm{H}}^{(s)}
$$

we can straightforwardly write (1) as

$$
\left\langle\Gamma\left(B_{s}(t) \rightarrow f\right)\right\rangle \propto e^{-\Gamma_{s} t}\left[\cosh \left(\frac{\Delta \Gamma_{s} t}{2}\right)+\mathcal{A}_{\Delta \Gamma}^{f} \sinh \left(\frac{\Delta \Gamma_{s} t}{2}\right)\right]
$$

with

$$
\mathcal{A}_{\Delta \Gamma}^{f} \equiv \frac{R_{\mathrm{H}}^{f}-R_{\mathrm{L}}^{f}}{R_{\mathrm{H}}^{f}+R_{\mathrm{L}}^{f}}
$$

We define the effective lifetime of the decay $B_{s}^{0} \rightarrow f$ as the time expectation value of the untagged rate [10],

$$
\tau_{f} \equiv \frac{\int_{0}^{\infty} t\left\langle\Gamma\left(B_{s}(t) \rightarrow f\right)\right\rangle d t}{\int_{0}^{\infty}\left\langle\Gamma\left(B_{s}(t) \rightarrow f\right)\right\rangle d t}=\frac{R_{\mathrm{L}}^{f} / \Gamma_{\mathrm{L}}^{(s) 2}+R_{\mathrm{H}}^{f} / \Gamma_{\mathrm{H}}^{(s) 2}}{R_{\mathrm{L}}^{f} / \Gamma_{\mathrm{L}}^{(s)}+R_{\mathrm{H}}^{f} / \Gamma_{\mathrm{H}}^{(s)}},
$$

which is equivalent to the lifetime that results from fitting the two exponentials in (1) to a single exponential [16]. By making the usual definition

$$
y_{s} \equiv \frac{\Delta \Gamma_{s}}{2 \Gamma_{s}},
$$

we can express the effective lifetime as

$$
\begin{aligned}
\frac{\tau_{f}}{\tau_{B_{s}}} & =\frac{1}{1-y_{s}^{2}}\left(\frac{1+2 \mathcal{A}_{\Delta \Gamma}^{f} y_{s}+y_{s}^{2}}{1+\mathcal{A}_{\Delta \Gamma}^{f} y_{s}}\right) \\
& =1+\mathcal{A}_{\Delta \Gamma}^{f} y_{s}+\left[2-\left(\mathcal{A}_{\Delta \Gamma}^{f}\right)^{2}\right] y_{s}^{2}+\mathcal{O}\left(y_{s}^{3}\right),
\end{aligned}
$$

where we have also given the expansion in powers of $y_{s}$ up to cubic corrections.

We proceed to consider the case where $f$ is a CP eigenstate with eigenvalue $\eta_{f}$. In the SM, the decay amplitude can be written, without loss of generality (using the unitarity of the Cabibbo-Kobayashi-Maskawa (CKM) matrix), as

$$
A\left(B_{s}^{0} \rightarrow f\right)=A_{1}^{f} e^{i \delta_{1}^{f}} e^{i \varphi_{1}^{f}}+A_{2}^{f} e^{i \delta_{2}^{f}} e^{i \varphi_{2}^{f}},
$$


where the $A_{1,2}^{f}$ are real and the $\delta_{1,2}^{f}$ and $\varphi_{1,2}^{f}$ are CP-conserving strong and CP-violating weak phases, respectively. Using the standard $B_{s}^{0}-\bar{B}_{s}^{0}$ mixing formalism [17], we have

$$
\mathcal{A}_{\Delta \Gamma}^{f}=\frac{2 \operatorname{Re} \xi_{f}^{(s)}}{1+\left|\xi_{f}^{(s)}\right|^{2}}
$$

where

$$
\xi_{f}^{(s)}=-\eta_{f} e^{-i \phi_{s}}\left[\frac{e^{-i \varphi_{1}^{f}}+h_{f} e^{i \delta_{f}} e^{-i \varphi_{2}^{f}}}{e^{i \varphi_{1}^{f}}+h_{f} e^{i \delta_{f}} e^{i \varphi_{2}^{f}}}\right] .
$$

Here we have introduced the abbreviation

$$
h_{f} e^{i \delta_{f}} \equiv \frac{A_{2}^{f}}{A_{1}^{f}} e^{i\left(\delta_{2}^{f}-\delta_{1}^{f}\right)},
$$

and $\phi_{s}$ denotes the $B_{s}^{0}-\bar{B}_{s}^{0}$ mixing phase, which is given by

$$
\phi_{s} \equiv \phi_{s}^{\mathrm{SM}}+\phi_{s}^{\mathrm{NP}}
$$

where $\phi_{s}^{\mathrm{SM}}$ and $\phi_{s}^{\mathrm{NP}}$ are the SM and NP pieces, respectively. It is convenient for the following discussion to introduce the direct $\mathrm{CP}$ asymmetry of the $B_{s} \rightarrow f$ decay [17]:

$$
C_{f} \equiv \frac{1-\left|\xi_{f}\right|^{2}}{1+\left|\xi_{f}\right|^{2}}=\frac{2 h_{f} \sin \delta_{f} \sin \left(\varphi_{1}^{f}-\varphi_{2}^{f}\right)}{N_{f}}
$$

where

$$
N_{f} \equiv 1+2 h_{f} \cos \delta_{f} \cos \left(\varphi_{1}^{f}-\varphi_{2}^{f}\right)+h_{f}^{2} .
$$

Subsequently, we may write

$$
\frac{2 \xi_{f}^{(s)}}{1+\left|\xi_{f}^{(s)}\right|^{2}}=-\eta_{f} \sqrt{1-C_{f}^{2}} e^{-i\left(\phi_{s}+\Delta \phi_{f}\right)} .
$$

Here $\Delta \phi_{f}$ is a hadronic phase shift, which is given by

$$
\begin{aligned}
\sin \Delta \phi_{f} & =\frac{\sin 2 \varphi_{1}^{f}+2 h_{f} \cos \delta_{f} \sin \left(\varphi_{1}^{f}+\varphi_{2}^{f}\right)+h_{f}^{2} \sin 2 \varphi_{2}^{f}}{N_{f} \sqrt{1-C_{f}^{2}}} \\
\cos \Delta \phi_{f} & =\frac{\cos 2 \varphi_{1}^{f}+2 h_{f} \cos \delta_{f} \cos \left(\varphi_{1}^{f}+\varphi_{2}^{f}\right)+h_{f}^{2} \cos 2 \varphi_{2}^{f}}{N_{f} \sqrt{1-C_{f}^{2}}},
\end{aligned}
$$

yielding

$$
\tan \Delta \phi_{f}=\frac{\sin 2 \varphi_{1}^{f}+2 h_{f} \cos \delta_{f} \sin \left(\varphi_{1}^{f}+\varphi_{2}^{f}\right)+h_{f}^{2} \sin 2 \varphi_{2}^{f}}{\cos 2 \varphi_{1}^{f}+2 h_{f} \cos \delta_{f} \cos \left(\varphi_{1}^{f}+\varphi_{2}^{f}\right)+h_{f}^{2} \cos 2 \varphi_{2}^{f}} .
$$

The twofold ambiguity for $\Delta \phi_{f}$ arising from the latter expression can be resolved using sign information from $\sin \Delta \phi_{f}$ or $\cos \Delta \phi_{f}$. These expressions generalize those given in Refs. [12, 18. 
Using (9) and (15), we thus obtain

$$
\mathcal{A}_{\Delta \Gamma}^{f}=-\eta_{f} \sqrt{1-C_{f}^{2}} \cos \left(\phi_{s}+\Delta \phi_{f}\right) .
$$

As we will see in Section 4 , there are fortunate $B_{s}$ decays into CP eigenstates where the hadronic parameter $h_{f} e^{i \delta_{f}}$ and the resulting phase shift $\Delta \phi_{f}$ can be controlled through experimental data. For these decays, we can hence use the corresponding lifetime measurements to constrain $y_{s}\left(\right.$ or $\left.\Delta \Gamma_{s}\right)$ with respect to $\phi_{s}$.

\section{Lifetime Contours in the $\phi_{s}-\Delta \Gamma_{s}$ Plane}

Let us now have a closer look at (7), which we can write as the following cubic equation for the real parameter $y_{s}$ :

$$
y_{s}^{3}+a_{2} y_{s}^{2}+a_{1} y_{s}+a_{0}=0
$$

where

$$
a_{0} \equiv \frac{\tau_{B_{s}}-\tau_{f}}{\tau_{f} \mathcal{A}_{\Delta \Gamma}^{f}}, \quad a_{1} \equiv \frac{2 \tau_{B_{s}}-\tau_{f}}{\tau_{f}}, \quad a_{2} \equiv \frac{\tau_{B_{s}}+\tau_{f}}{\tau_{f} \mathcal{A}_{\Delta \Gamma}^{f}} .
$$

In order to solve this cubic equation, it is useful to rewrite it in the "reduced" form

$$
\left(y_{s}+\frac{a_{2}}{3}\right)^{3}+P\left(y_{s}+\frac{a_{2}}{3}\right)+Q=0
$$

with

$$
P \equiv a_{1}-\frac{a_{2}^{2}}{3}, \quad Q \equiv \frac{2 a_{2}^{3}}{27}-\frac{a_{2} a_{1}}{3}+a_{0}
$$

Applying Cardano's formula then yields the solutions

$$
y_{s}=-\frac{a_{2}}{3}+e^{i \omega} \sqrt[3]{R+\sqrt{D}}+e^{-i \omega} \sqrt[3]{R-\sqrt{D}}
$$

with $\omega \in\{0,2 \pi / 3,-2 \pi / 3\}$, where

$$
\begin{gathered}
R \equiv-\frac{Q}{2}=\frac{1}{54}\left(9 a_{1} a_{2}-27 a_{0}-2 a_{2}^{3}\right) \\
D \equiv\left(\frac{P}{3}\right)^{3}+\left(\frac{Q}{2}\right)^{2}=\frac{1}{108}\left(27 a_{0}^{2}-18 a_{0} a_{1} a_{2}+4 a_{0} a_{2}^{3}+4 a_{1}^{3}-a_{1}^{2} a_{2}^{2}\right) .
\end{gathered}
$$

For $\mathcal{A}_{\Delta \Gamma}^{f}=0$, this solution is not valid as 77 is then a quadratic equation in $y_{s}$. Furthermore, the above expressions may prove cumbersome to use in practice. A convenient approximate solution is obtained by solving the expansion in (7) up to quadratic order in $y_{s}$ :

$$
y_{s} \approx-\frac{1}{2}\left[\frac{\mathcal{A}_{\Delta \Gamma}^{f}}{2-\left(\mathcal{A}_{\Delta \Gamma}^{f}\right)^{2}}\right] \pm \frac{1}{2} \sqrt{\left[\frac{\mathcal{A}_{\Delta \Gamma}^{f}}{2-\left(\mathcal{A}_{\Delta \Gamma}^{f}\right)^{2}}\right]^{2}+\frac{4}{\tau_{B_{s}}}\left[\frac{\tau_{f}-\tau_{B_{s}}}{2-\left(\mathcal{A}_{\Delta \Gamma}^{f}\right)^{2}}\right]}
$$




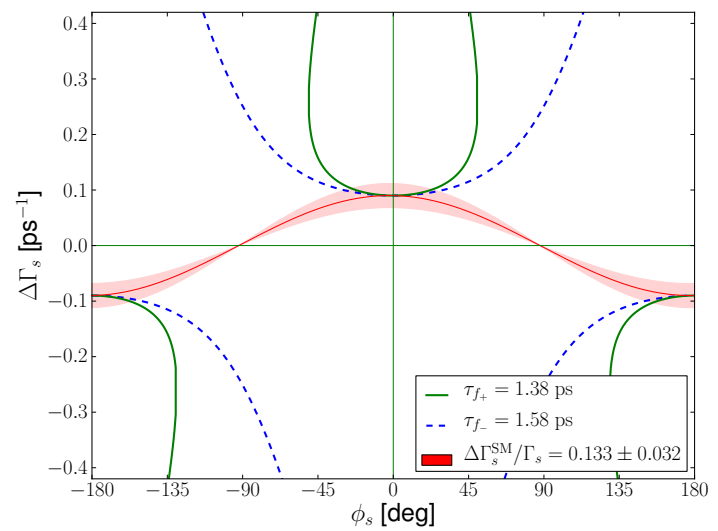

Figure 1: Illustration of the lifetimes that are compatible with the SM value of $\Delta \Gamma_{s} / \Gamma_{s}$ given in $(29)$ for $\mathrm{CP}$-even and $\mathrm{CP}$-odd final states $f_{+}$and $f_{-}$, respectively. The decay amplitudes are assumed to have no $\mathrm{CP}$-violating phases. We also show the constraint from the theoretical value of $\Delta \Gamma_{s}^{\mathrm{SM}} / \Gamma_{s}$ given in 29$]$, as discussed in the text.

This quadratic solution is in excellent agreement with the corresponding branches of the exact solution (24) for the numerical analyses discussed below.

For illustration we consider two $B_{s}$ decays to CP eigenstates, $B_{s} \rightarrow f_{+}$and $B_{s} \rightarrow f_{-}$, with positive and negative $\mathrm{CP}$ eigenvalues, respectively. Further, we assume

$$
h_{f_{ \pm}}=0, \quad \varphi_{1}^{f_{ \pm}}=0
$$

for these decays, yielding $C_{f_{ \pm}}=0$ and $\Delta \phi_{f_{ \pm}}=0$. In Fig. 1. we show the lifetime constraints that are compatible with the theoretical SM calculation of $\Delta \Gamma_{s}$ [19],

$$
\frac{\Delta \Gamma_{s}^{\mathrm{SM}}}{\Gamma_{s}}=2 y_{s}^{\mathrm{SM}}=0.133 \pm 0.032,
$$

and the SM value of the $B_{s}^{0}-\bar{B}_{s}^{0}$ mixing phase, which is given as follows [20]:

$$
\phi_{s}^{\mathrm{SM}} \equiv-2 \beta_{s}=-(2.08 \pm 0.09)^{\circ} .
$$

Throughout this paper, we shall use 21

$$
\tau_{B_{s}}=\left(1.477_{-0.022}^{+0.021}\right) \mathrm{ps}
$$

for the $B_{s}$ lifetime introduced in (2), resulting in the SM effective lifetimes $\tau_{f_{+}}=1.38 \mathrm{ps}$ and $\tau_{f_{-}}=1.58 \mathrm{ps}$. The difference in behaviour for CP-odd and CP-even eigenstates is due to the non-linear dependence on $y_{s}$ in (7). Said differently, if (7) is expanded and only terms up to linear order in $y_{s}$ are kept the two curves in Fig. 1 would overlap.

In Fig. 1. we have included another constraint, which is related to the theoretical value in 29] as follows: if we assume that NP can only affect $\Delta \Gamma_{s}$ through $B_{s}^{0}-\bar{B}_{s}^{0}$ mixing, which is a very plausible assumption, we have 22

$$
y_{s}=\frac{\Delta \Gamma_{s}^{\mathrm{SM}} \cos \tilde{\phi}_{s}}{2 \Gamma_{s}}=y_{s}^{\mathrm{SM}} \cos \tilde{\phi}_{s},
$$


where

$$
\tilde{\phi}_{s} \equiv \tilde{\phi}_{s}^{\mathrm{SM}}+\phi_{s}^{\mathrm{NP}}
$$

Here $\phi_{s}^{\mathrm{NP}}$ is the NP $B_{s}^{0}-\bar{B}_{s}^{0}$ mixing phase, which also enters $\phi_{s}$ defined in 12 on which $\mathcal{A}_{\Delta \Gamma}^{f}$ depends, whereas the SM piece takes the following value [19]:

$$
\tilde{\phi}_{s}^{\mathrm{SM}}=(0.22 \pm 0.06)^{\circ} .
$$

The formalism developed above is also valid for non-CP eigenstates provided the final state is accessible to both $B_{s}^{0}$ and $\bar{B}_{s}^{0}$ so that mixing is possible. Examples of such states are $B_{s} \rightarrow D_{s}^{ \pm} K^{(*) \mp}$. For these decays the CP eigenvalue $\eta_{f}$ in (19) should be replaced by $(-1)^{L}$, where $L$ denotes the relative orbital angular momentum of the decay products 23$]$.

\section{Hadronic Corrections and Their Control}

Examples of effective lifetimes that have been measured for $B_{s}$ decays to CP-even and $\mathrm{CP}$-odd final states are $B_{s}^{0} \rightarrow K^{+} K^{-}$and $B_{s}^{0} \rightarrow J / \psi f_{0}$, respectively. Unlike our hypothetical examples from the previous section, however, the decay amplitudes of these decays are not devoid of weak phases, and can be written in the SM as follows [9, 10,12]:

$$
\begin{gathered}
A\left(B_{s}^{0} \rightarrow K^{+} K^{-}\right)=\lambda \mathcal{C}\left[e^{i \gamma}+\frac{1}{\epsilon} d e^{i \theta}\right] \\
A\left(B_{s}^{0} \rightarrow J / \psi f_{0}\right)=\left(1-\frac{\lambda^{2}}{2}\right) \mathcal{A}\left[1+\epsilon b e^{i \vartheta} e^{i \gamma}\right] .
\end{gathered}
$$

Here $\lambda \equiv\left|V_{u s}\right|=0.2252 \pm 0.0009$ is the Wolfenstein parameter of the CKM matrix [24],

$$
\epsilon \equiv \frac{\lambda^{2}}{1-\lambda^{2}}=0.0534 \pm 0.0005
$$

and $\gamma$ is the usual angle of the unitarity triangle whereas $\mathcal{C}, d e^{i \theta}$ and $\mathcal{A}, b e^{i \theta}$ are hadronic, CP-conserving parameters.

Consequently, the parameters introduced in (9) and (11) take the forms

$$
h_{K^{+} K^{-}}=d / \epsilon, \quad \delta_{K^{+} K^{-}}=\theta, \quad \varphi_{1}^{K^{+} K^{-}}=\gamma, \quad \varphi_{2}^{K^{+} K^{-}}=0
$$

and

$$
h_{J / \psi f_{0}}=\epsilon b, \quad \delta_{J / \psi f_{0}}=\vartheta, \quad \varphi_{1}^{J / \psi f_{0}}=0, \quad \varphi_{2}^{J / \psi f_{0}}=\gamma,
$$

so that the hadronic phase shifts can be obtained from

$$
\begin{aligned}
& \tan \Delta \phi_{K^{+} K^{-}}=2 \epsilon\left[\frac{d \cos \theta+\epsilon \cos \gamma}{d^{2}+2 \epsilon d \cos \theta \cos \gamma+\epsilon^{2} \cos 2 \gamma}\right] \sin \gamma \\
& \tan \Delta \phi_{J / \psi f_{0}}=2 \epsilon b\left[\frac{\cos \vartheta+\epsilon b \cos \gamma}{1+2 \epsilon b \cos \vartheta \cos \gamma+\epsilon^{2} b^{2} \cos 2 \gamma}\right] \sin \gamma
\end{aligned}
$$


We observe that these phases are proportional to the tiny $\epsilon$ parameter, i.e. are doubly Cabibbo-suppressed. Consequently, the $\mathcal{A}_{\Delta \Gamma}^{f}$ observable given in 19 is robust with respect to hadronic uncertainties for the decays at hand.

In the case of the $B_{s}^{0} \rightarrow K^{+} K^{-}$channel, we can use the $U$-spin symmetry of strong interactions to relate it to the $B_{d}^{0} \rightarrow \pi^{+} \pi^{-}$decay, which thereby allows us to determine $\gamma$ as well as $d$ and $\theta[9]$. The current status of an analysis along these lines, using also the direct $\mathrm{CP}$ violation in $B_{d} \rightarrow \pi^{\mp} K^{ \pm}$, is given by [10]:

$$
\gamma=(68 \pm 7)^{\circ}, \quad d=0.50_{-0.11}^{+0.12}, \quad \theta=\left(154_{-14}^{+11}\right)^{\circ},
$$

where the errors include the uncertainties of the relevant input quantities and estimates of $U$-spin-breaking corrections. The $\gamma$ result is in excellent agreement with the current fits of the unitarity triangle [25, 26], thereby excluding large CP-violating NP contributions to the $B_{s}^{0} \rightarrow K^{+} K^{-}$decay amplitude. Using these numbers in 400 , we find

$$
\Delta \phi_{K^{+} K^{-}}=-\left(\left.\left.\left.10.5_{-0.5}^{+0.3}\right|_{\gamma^{-2.1}} ^{+2.9}\right|_{d^{-1.7}} ^{+0.9}\right|_{\theta}\right)^{\circ}=-\left(10.5_{-2.8}^{+3.1}\right)^{\circ},
$$

where we have added the errors in quadrature. Similarly, we also find $C_{K^{+} K^{-}}=0.09_{-0.04}^{+0.05}$.

Unfortunately, as discussed in Ref. [12], it is much more involved to control the hadronic effects in the $B_{s}^{0} \rightarrow J / \psi f_{0}$ decay through experimental data, and the potential control channel $B_{d}^{0} \rightarrow J / \psi f_{0}$ has not yet been observed. On the other hand, contrary to $B_{s}^{0} \rightarrow K^{+} K^{-}$, the denominator of (41) is equal to one at leading order in $\epsilon$. Following Ref. [12], we use the conservative range $0 \leq b \leq 0.5$ and leave $\vartheta$ unconstrained. Using moreover the value for $\gamma$ in $(42)$, we find

$$
\Delta \phi_{J / \psi f_{0}} \in\left[-2.9^{\circ}, 2.8^{\circ}\right]
$$

and $\left|C_{J / \psi f_{0}}\right| \lesssim 0.05$, which has, just like $C_{K^{+} K^{-}}$, a negligible impact on 19 .

It is remarkable that the hadronic phase shifts $\Delta \phi_{K^{+} K^{-}}$and $\Delta \phi_{J / \psi f_{0}}$ turn out to be so robust with respect to the hadronic effects and the weak phase $\gamma$, suffering from uncertainties of only $\sim 3^{\circ}$. Future data should allow us to determine them with even higher precision.

\section{Constraints from Current and Future Data}

In the previous section we presented all the ingredients necessary to compute $\mathcal{A}_{\Delta \Gamma}^{K^{+} K^{-}}$and $\mathcal{A}_{\Delta \Gamma}^{J / \psi f_{0}}$ as functions of $\phi_{s}$. Inserting them into the solution for $y_{s}$ discussed in Section 3 allows us to draw contours for the lifetime measurements on the $\phi_{s}-\Delta \Gamma_{s}$ plane. The first measurement of the effective $B_{s}^{0} \rightarrow K^{+} K^{-}$lifetime was performed by the CDF collaboration in 2006 [13]. In the spring of 2011, the LHCb collaboration reported their first measurement of this observable [14]:

$$
\tau_{K^{+} K^{-}}=[1.44 \pm 0.096(\text { stat }) \pm 0.010(\text { syst })] \mathrm{ps},
$$

which is currently the most precise. The first measurement of the $B_{s}^{0} \rightarrow J / \psi f_{0}$ lifetime has recently been made by the CDF collaboration [15]:

$$
\tau_{J / \psi f_{0}}=\left[1.70_{-0.11}^{+0.12}(\text { stat }) \pm 0.03(\text { syst })\right] \text { ps. }
$$



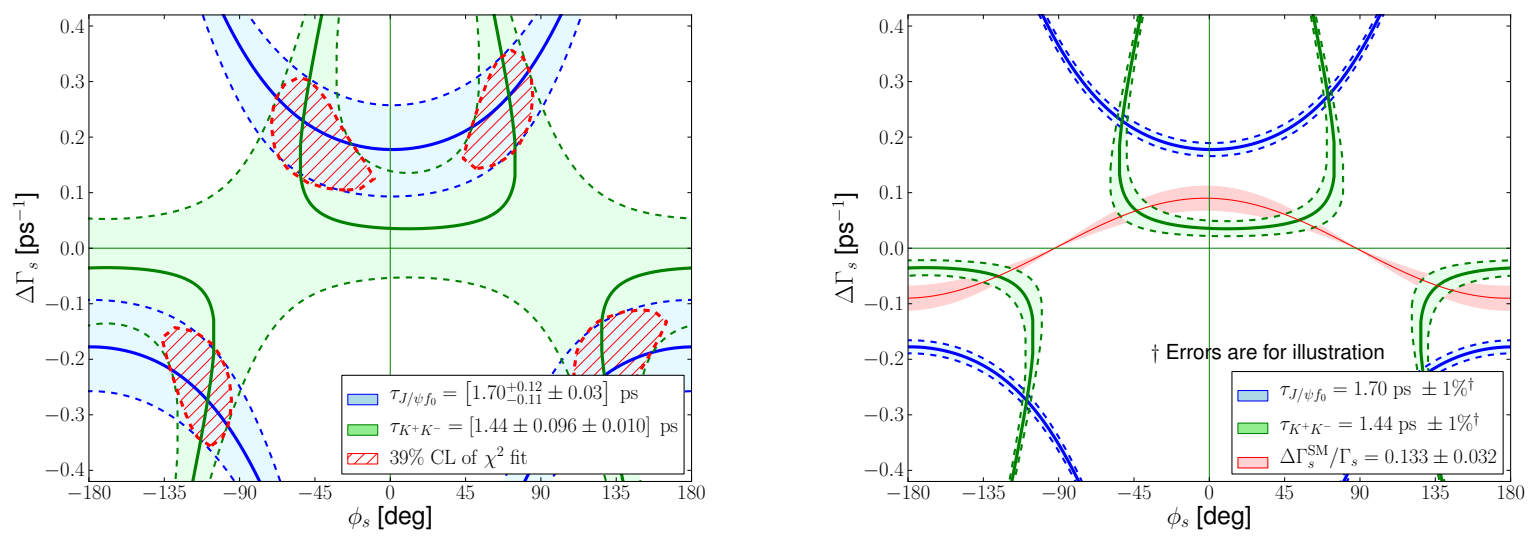

Figure 2: The measurements of the effective $B_{s}^{0} \rightarrow K^{+} K^{-}$and $B_{s}^{0} \rightarrow J / \psi f_{0}$ lifetimes projected onto the $\phi_{s}-\Delta \Gamma_{s}$ plane. Left panel: analysis of the current data, where the shaded bands give the $1 \sigma$ uncertainties of the lifetimes; the $39 \%$ confidence regions originating from a $\chi^{2}$ fit are also shown. Right panel: illustration of how the situation improves for unchanged central values if the uncertainties were improved to $1 \%$ accuracy, including also the constraint from the theoretical value of $\Delta \Gamma_{s}^{\mathrm{SM}} / \Gamma_{s}$.

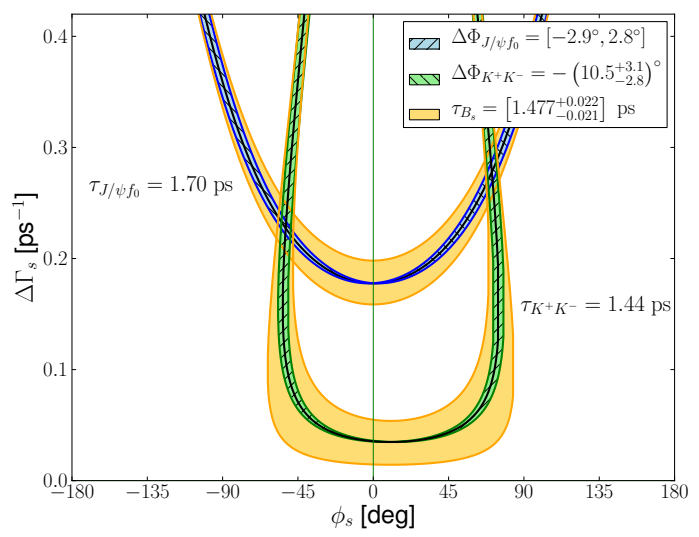

Figure 3: Illustration of the errors of the hadronic phase shifts $\Delta \phi_{K^{+} K^{-}}$and $\Delta \phi_{J / \psi f_{0}}$ on the contours in the $\phi_{s}-\Delta \Gamma_{s}$ plane for the central values of the lifetime measurements. We also shown the impact of the present error of the $B_{s}$ lifetime. 


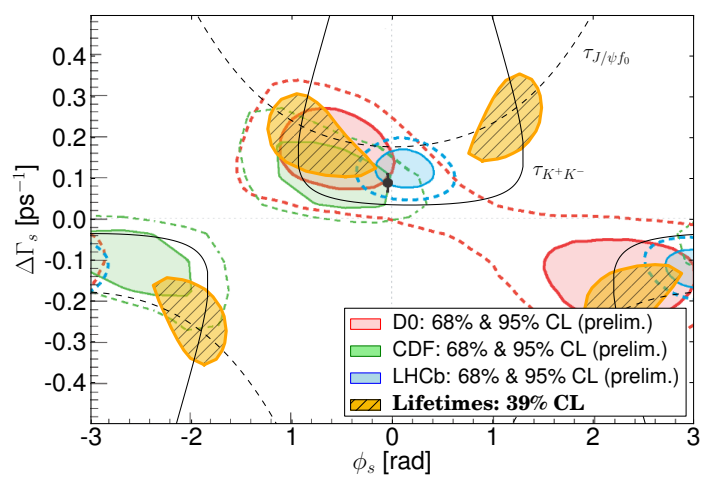

Figure 4: The fitted lifetime regions in the $\phi_{s}-\Delta \Gamma_{s}$ plane from the left panel of Fig. 2 added to a compilation of measurements as obtained in Ref. [29]. The DØ, CDF and $\mathrm{LHCb}$ allowed regions refer to tagged analyses of $B_{s}^{0} \rightarrow J / \psi \phi$. In addition, the D $\varnothing$ region includes also the result for the like-sign dimuon asymmetry while LHCb has also included a first analysis of $\mathrm{CP}$ violation in $B_{s}^{0} \rightarrow J / \psi f_{0}$.

In the left panel of Fig. 2, we show the current measurements of the effective lifetimes of the $B_{s}^{0} \rightarrow K^{+} K^{-}$and $B_{s}^{0} \rightarrow J / \psi f_{0}$ decays as constraints on the $\phi_{s}-\Delta \Gamma_{s}$ plane. We also show the $39 \%$ confidence region resulting from a $\chi^{2}$ fit of these two results. The individual fitted values for the $\phi_{s}$ and $\Delta \Gamma_{s}$ parameters are given as follows:

$$
\begin{gathered}
\phi_{s}=-\left(52_{-43}^{+19}\right)^{\circ}, \quad \Delta \Gamma_{s}=\left(0.23_{-0.12}^{+0.08}\right) \mathrm{ps}^{-1} \\
\phi_{s}=\left(71_{-27}^{+14}\right)^{\circ}, \quad \Delta \Gamma_{s}=\left(0.28_{-0.14}^{+0.08}\right) \mathrm{ps}^{-1}
\end{gathered}
$$

where the errors are $68 \%$ confidence levels corresponding to a $\chi^{2}$ fit of the lifetimes. Each solution has a two-fold ambiguity given by the transformation

$$
\phi_{s} \rightarrow \phi_{s}+180^{\circ}, \quad \Delta \Gamma_{s} \rightarrow-\Delta \Gamma_{s} .
$$

Both lifetime measurements currently have an error of about $7 \%$. However, it seems feasible to reduce the uncertainty of the $\tau_{K^{+} K^{-}}$measurement at LHCb to the few-percent level [27]. In the right panel of Fig. 2, we show - for illustration - the impact of measurements of the $B_{s}^{0} \rightarrow K^{+} K^{-}$and $B_{s}^{0} \rightarrow J / \psi f_{0}$ lifetimes with $1 \%$ uncertainty, assuming no change in the central values. Clearly, at this level of accuracy, the lifetime measurements could strongly constrain $\phi_{s}$ and $\Delta \Gamma_{s}$.

Using (32), we also include the band corresponding to the theoretical value of $\Delta \Gamma_{s}^{\mathrm{SM}} / \Gamma_{s}$ given in 290 . We observe, as also noted in Ref. [12], that the central value of the $\tau_{J / \psi f_{0}}$ measurement is too large in comparison with this constraint. To spoil the relation in (32) either large NP effects are required, a very contrived scenario in our opinion, or the width difference $\Delta \Gamma_{s}$ must be affected by hadronic long-distance effects, which are not included in the SM calculation of (29). The $B_{s}^{0} \rightarrow J / \psi f_{0}$ effective lifetime predicted by the SM calculation is $\tau_{J / \psi f_{0}}=(1.582 \pm 0.036)$ ps $[12]$. 
The uncertainties of the hadronic phase shifts given in (43) and (44) as well as the error of the $B_{s}$ lifetime in (31) were not included in Fig. 2 or in the fit results in (47) and 48). In Fig. 3, we illustrate the impact of these uncertainties on the lifetime contours in the $\phi_{s}-\Gamma_{s}$ plane. Comparing with the error bands in Fig. 2, we observe that the effects of these uncertainties are marginal with respect to the current errors of the effective lifetime measurement. More sophisticated fits should take these uncertainties into account as well.

It is interesting to compare our fitted results to recent measurements of $\mathrm{CP}$ violation in the $B_{s}^{0} \rightarrow J / \psi \phi$ channel, where the current picture of $\phi_{s}$ looks as follows: CDF finds $\phi_{s} \in\left[-177.6^{\circ},-123.8^{\circ}\right] \vee\left[-59.6^{\circ},-2.3^{\circ}\right](68 \%$ C.L. $)$, while $\mathrm{D} \varnothing$ has recently reported $\phi_{s}=-\left(31.5_{-21.8}^{+20.6}\right)^{\circ}[6$. The LHCb collaboration has also entered the arena, reporting $\phi_{s}=+(7.4 \pm 10.3 \pm 4.0)^{\circ}$ and $\Delta \Gamma_{s}=[0.123 \pm 0.029$ (stat) \pm 0.008 (syst) $] \mathrm{ps}^{-1}$ [8]. Furthermore, LHCb has presented a first tagged analysis of the CP-violating asymmetry of the $B_{s}^{0} \rightarrow J / \psi f_{0}$ channel, yielding $\phi_{s}=-(25 \pm 25 \pm 1)^{\circ}[28]$.

A compilation of the preliminary results from the $\mathrm{D} \varnothing, \overline{\mathrm{CDF}}$ and $\mathrm{LHCb}$ collaborations as constraints in the $\phi_{s}-\Delta \Gamma_{s}$ plane has recently been performed in Ref. [29]. In Fig. 4 , we have overlaid on the corresponding plot the lifetime contours and fit results of the analysis described above. It is intriguing to see how well the lifetime allowed region overlaps with those from $\mathrm{D} \varnothing$ and CDF.

The current errors leave space for interesting future developments. Should the central values of the $\mathrm{CP}$-even and $\mathrm{CP}$-odd lifetimes approach the theoretical SM point, the power to pinpoint $\phi_{s}$ is lost, as illustrated by Fig. 1. However, because the curves are flat at this point, $\Delta \Gamma_{s}$ could still be determined accurately in this case.

\section{Further Promising $\boldsymbol{B}_{s}$ Decays}

So far we have discussed the effective lifetimes for the $B_{s}^{0} \rightarrow K^{+} K^{-}$and $B_{s}^{0} \rightarrow J / \psi f_{0}$ channels, which have both been measured in first analyses by the CDF and LHCb collaborations. These channels have final states with opposite CP eigenvalues and happen to be well paired for obtaining constraints in the $\phi_{s}-\Delta \Gamma_{s}$ plane using the strategy proposed in this paper. The hadronic corrections in $B_{s}^{0} \rightarrow K^{+} K^{-}$can be controlled even better in the future through precise measurements of the CP-violating observables of the $B_{d}^{0} \rightarrow \pi^{+} \pi^{-}$channel. Regarding $B_{s}^{0} \rightarrow J / \psi f_{0}$, hadronic corrections have a minor impact on the lifetime analysis. A potential control channel is $B_{d}^{0} \rightarrow J / \psi f_{0}$, although here the situation is much more involved than in $B_{s}^{0} \rightarrow K^{+} K^{-}$due to the unsettled hadronic structure of the scalar $f_{0}(980)$ state $[12]$.

Another interesting decay that can soon be added to this picture is $B_{s}^{0} \rightarrow J / \psi K_{\mathrm{S}}$ 30, 31, which has been observed by the CDF and LHCb collaborations 32, 33. This channel has a final state with $\mathrm{CP}$ eigenvalue -1 and is caused by $\bar{b} \rightarrow \bar{c} c \bar{c}$ quark-level processes, i.e. it has a CKM structure that is different from the decays considered above. In particular, the relevant hadronic parameter does not enter in a doubly Cabibbosuppressed way. However, the uncertainties can be controlled through $B_{d}^{0} \rightarrow J / \psi \pi^{0}$ and are found to have a moderate impact on the effective $B_{s}^{0} \rightarrow J / \psi K_{\mathrm{S}}$ lifetime [34], which has not yet been measured. 
Another $B_{s}$ decay with a CP-even final state is $B_{s}^{0} \rightarrow D_{s}^{+} D_{s}^{-}$. Here the hadronic corrections are again doubly Cabibbo-suppressed and can be controlled with the help of the $U$-spin-related $B_{d}^{0} \rightarrow D^{+} D^{-}$decay [30]. A first theoretical analysis of the effective lifetime of $B_{s}^{0} \rightarrow D_{s}^{+} D_{s}^{-}$was performed in Ref. [35]. Further decays into CP-even final states where a similar analysis can be performed are the $B_{s(d)}^{0} \rightarrow J / \psi \eta^{\left({ }^{\prime}\right)}$ channels [36].

Decays of $B_{s}$ mesons into CP-selfconjugate final states with two vector mesons or higher resonances offer another laboratory for lifetime analyses. In this case the $B_{s}^{0} \rightarrow \phi \phi$ and $B_{s}^{0} \rightarrow K^{* 0} \bar{K}^{* 0}$ channels look particularly interesting. These decays, which have already been observed experimentally [6, 8, 37], are penguin modes. Their final states are mixtures of CP-even and $\mathrm{CP}$-odd eigenstates and can be disentangled by means of angular analyses. It would be interesting to perform measurements of the lifetimes for the CP-even and CP-odd final-state configurations and to add them as contours to the $\phi_{s}-\Gamma_{s}$ plane along the lines of the strategy proposed above.

A similar comment applies to the $B_{s} \rightarrow J / \psi \phi$ channel, where it would also be desirable to determine the individual lifetimes for the CP-even and CP-odd final-state configurations separately instead of making a fit to the whole time-dependent angular distribution. This can be done by means of the moment analysis proposed in Ref. [3]. The hadronic uncertainties of the $B_{s}^{0} \rightarrow J / \psi \phi$ channel can be controlled by channels such as $B_{s}^{0} \rightarrow J / \psi \bar{K}^{* 0}$ and $B_{d}^{0} \rightarrow J / \psi \rho^{0}[18]$.

In the future, also decays with final states that are not CP-selfconjugate can be added to the agenda to further constrain $\phi_{s}$ and $\Delta \Gamma_{s}$, provided both a $B_{s}^{0}$ and a $\bar{B}_{s}^{0}$ meson can decay into the same final state (see Section 2). Prime examples are the $B_{s} \rightarrow D_{s}^{ \pm} K^{(*) \mp}$ channels. Their effective lifetimes can be used to constrain $\phi_{s}+\gamma$ with respect to $\Delta \Gamma_{s}$ (see Ref. 23] for an overview of the observables of these decays).

\section{Conclusions}

Thanks to the sizable width difference $\Delta \Gamma_{s}$ of the $B_{s}$-meson system, effective lifetimes of $B_{s}$ decays offer interesting probes of $B_{s}^{0}-\bar{B}_{s}^{0}$ mixing. The corresponding measurements require only untagged data samples and are advantageous from an experimental point of view. Thanks to non-linear terms in $\Delta \Gamma_{s}$, a pair of $B_{s}$ decays into CP-even and CP-odd final states is sufficient to determine the $B_{s}^{0}-\bar{B}_{s}^{0}$ mixing phase and the width difference. Prime examples for implementing this strategy in practice are the decays $B_{s}^{0} \rightarrow K^{+} K^{-}$and $B_{s}^{0} \rightarrow J / \psi f_{0}$. Their effective lifetimes turn out to be robust with respect to hadronic uncertainties, which can be controlled or constrained with the help of further experimental data.

We have calculated the constraints in the $\phi_{s}-\Delta \Gamma_{s}$ plane following from the current measurements of the $B_{s}^{0} \rightarrow K^{+} K^{-}$and $B_{s}^{0} \rightarrow J / \psi f_{0}$ lifetimes, which both suffer from $\sim 7 \%$ uncertainties. The resulting picture is consistent with other constraints following from Tevatron measurements, which have not been supported by recent LHCb data. The uncertainties still preclude us from drawing definite conclusions but leave space for interesting future developments. Lifetime measurements with $1 \%$ precision would allow us to obtain much stronger constraints in the $\phi_{s}-\Delta \Gamma_{s}$ plane as we have illustrated in our study. Should the central values of the lifetimes for the $\mathrm{CP}$-even and $\mathrm{CP}$-odd final states 
approach the SM values, the lifetime contours would loose their power to determine $\phi_{s}$. However, the width difference could still be determined in a precise way in this case.

An interesting trend of the current data is that it favours a value of $\Delta \Gamma_{s}$ that is larger than the one calculated in QCD. With the plausible assumption that NP affects this observable only through $B_{s}^{0}-\bar{B}_{s}^{0}$ mixing, its absolute value can only decrease and hence the discrepancy will be even larger for sizable mixing phases. This feature raises the question of whether the SM calculation of $\Delta \Gamma_{s}$ fully includes all hadronic longdistance contributions. It will be interesting to see if this trend will be supported by future data or if it will eventually disappear.

In the future, also other effective lifetime measurements of $B_{s}$-meson decays can be added to the $\phi_{s}-\Delta \Gamma_{s}$ plane, allowing us to "overconstrain" the mixing parameters in the same spirit as the determination of the apex of the unitarity triangle. This information will be complementary to the tagged analyses of $\mathrm{CP}$ violation in the $B_{s}$ system. It will be intriguing to see at which point of the $\phi_{s}-\Delta \Gamma_{s}$ plane all measurements will eventually converge.

\section{References}

[1] P. Ball and R. Fleischer, Eur. Phys. J. C 48 (2006) 413 arXiv:hep-ph/0604249; Z. Ligeti, M. Papucci and G. Perez, Phys. Rev. Lett. 97 (2006) 101801 |hepph/0604112]; J. L. Rosner and M. Gronau, arXiv:1105.1923 [hep-ph]; A. J. Buras, arXiv:1106.0998 [hep-ph].

[2] I. Dunietz, R. Fleischer and U. Nierste, Phys. Rev. D 63 (2001) 114015 arXiv:hepph/0012219.

[3] A. S. Dighe, I. Dunietz and R. Fleischer, Eur. Phys. J. C 6 (1999) 647 arXiv:hepph/9804253.

[4] F. Azfar et al. [CDF Collaboration], Public Note 10206 (2010).

[5] V. M. Abazov et al. [D $\varnothing$ Collaboration], arXiv:1109.3166 [hep-ex].

[6] R. Van Kooten, talk at Lepton-Photon 2011, Mumbai, India, 22-27 August 2011 http://www.tifr.res.in/ lp11/].

[7] V. M. Abazov et al. [DØ Collaboration], arXiv:1106.6308 [hep-ex].

[8] G. Raven, talk at Lepton-Photon 2011, Mumbai, India, 22-27 August 2011 http://www.tifr.res.in/ lp11/].

[9] R. Fleischer, Phys. Lett. B 459 (1999) 306 arXiv:hep-ph/9903456; Eur. Phys. J. C 52 (2007) 267 arXiv:0705.1121 [hep-ph]].

[10] R. Fleischer and R. Knegjens, Eur. Phys. J. C 71 (2011) 1532 arXiv:1011.1096 [hep-ph]]. 
[11] S. Stone and L. Zhang, Phys. Rev. D 79 (2009) 074024 [arXiv:0812.2832 [hep-ph]]; arXiv:0909.5442 [hep-ex].

[12] R. Fleischer, R. Knegjens and G. Ricciardi, arXiv:1109.1112 [hep-ph].

[13] CDF collaboration, CDF Note 06-01-26 (2006).

[14] L. Eklund et al. [LHCb Collaboration], LHCb-CONF-2011-018.

[15] T. Aaltonen et al. [CDF Collaboration], arXiv:1106.3682 [hep-ex].

[16] K. Hartkorn and H. G. Moser, Eur. Phys. J. C 8 (1999) 381.

[17] R. Fleischer, Phys. Rept. 370 (2002) 537 arXiv:hep-ph/0207108.

[18] S. Faller, R. Fleischer and T. Mannel, Phys. Rev. D 79 (2009) 014005 arXiv:0810.4248 [hep-ph]].

[19] A. Lenz and U. Nierste, arXiv:1102.4274 [hep-ph]; A. Lenz and U. Nierste, JHEP 0706 (2007) 072 arXiv:hep-ph/0612167].

[20] J. Charles et al., arXiv:1106.4041 [hep-ph].

[21] D. Asner et al. [Heavy Flavor Averaging Group], arXiv:1010.1589 [hep-ex], see also http://www.slac.stanford.edu/xorg/hfag/

[22] Y. Grossman, Phys. Lett. B 380 (1996) 99 arXiv:hep-ph/9603244.

[23] R. Fleischer, Nucl. Phys. B 671 (2003) 459 arXiv:hep-ph/0304027.

[24] K. Nakamura et al. [Particle Data Group], J. Phys. G 37 (2010) 075021.

[25] CKMfitter Collaboration, http://ckmfitter.in2p3.fr/

[26] UTfit Collaboration, http://www.utfit.org/UTfit/WebHome

[27] M. Gersabeck, private communication and CERN-THESIS-2009-118 (2009).

[28] B. Pal et al. [LHCb Collaboration], LHCb-CONF-2011-051.

[29] LHCb Collaboration, public website, 27 August 2011 http://lhcb-public.web.cern.ch/lhcb-public/

[30] R. Fleischer, Eur. Phys. J. C 10 (1999) 299 arXiv:hep-ph/9903455.

[31] K. De Bruyn, R. Fleischer and P. Koppenburg, Eur. Phys. J. C 70 (2010) 1025 arXiv:1010.0089 [hep-ph]];

[32] T. Aaltonen et al. [CDF Collaboration], Phys. Rev. D 83 (2011) 052012 arXiv:1102.1961 [hep-ex]].

[33] P. Koppenburg et al. [LHCb Collaboration], LHCb-CONF-2011-048. 
[34] K. De Bruyn, R. Fleischer and P. Koppenburg, Proceedings of CKM 2010, Warwick, United Kingdom, 6-10 September 2010, arXiv:1012.0840 [hep-ph].

[35] R. Fleischer, Eur. Phys. J. C 51 (2007) 849 arXiv:0705.4421 [hep-ph]].

[36] P. Z. Skands, JHEP 0101 (2001) 008 arXiv:hep-ph/0010115].

[37] B. Adeva et al. [LHCb Collaboration], LHCb-CONF-2011-019. 